\title{
All-fiber High Repetition Rate Short Pulse Generation around 1030nm
}

\author{
Qin Li $^{\mathrm{a}}$, Rui Zhu ${ }^{\mathrm{a}}$,Chi Zhang ${ }^{\mathrm{a}}$, Sigang Yang ${ }^{\mathrm{b}}$ and Kenneth K. Y. Wong ${ }^{* a}$ \\ ${ }^{a}$ Photonic Systems Research Laboratory, Department of Electrical and Electronic Engineering, \\ The University of Hong Kong, Hong Kong; \\ ${ }^{b}$ Department of Electronic Engineering, Tsinghua University, Beijing, China \\ *Email: kywong@eee.hku.hk
}

\begin{abstract}
High repetition rate pulsed fiber laser in $1 \mu \mathrm{m}$ is an attractive and novel source for optical transmission systems, since ytterbium-doped fiber (YDF) has the potential to provide broad gain spectrum and high optical conversion efficiency in this regime. Previous works in this area have explored the wavelength range above $1050 \mathrm{~nm}$. In this paper, we focus more on the shorter wavelength band which is closer to the peak of the emission cross section of YDF at around $1030 \mathrm{~nm}$. A 10-GHz harmonically mode-locked all-fiber laser is demonstrated. A pulse train with a pulsewidth of around $13 \mathrm{ps}$ and wavelength tunable from $1023.5 \mathrm{~nm}$ to $1053.3 \mathrm{~nm}$ is achieved. The side-mode suppression ratio is more than $50 \mathrm{~dB}$ without any stabilization techniques.
\end{abstract}

Keywords: fiber lasers; active mode locking; ytterbium-doped fiber

\section{INTRODUCTION}

With the unprecedented development of the fiber technology, YDF-based fiber lasers are becoming real competitors to the ytterbium-based bulk lasers. YDF is an ideal fiber gain medium for ultrashort pulse generation in 1- $\mu \mathrm{m}$ band since it has broad gain bandwidth, high doping level, and high optical conversion efficiency ${ }^{1}$. An all-fiber laser setup is competitive for its low loss, free of maintenance, and compatibility with other fiber systems. Mode-locking techniques, either passive or active, are the dominant methods in the generation of pulsed lasers. Many works about the pulsed fiber laser based on YDF used the passive mode-locking technologies ${ }^{2,3}$. Ultrashort pulsewidth ${ }^{2}$ and wide tuning range ${ }^{3}$ have been reported. However, most of the passive mode-locking lasers were mode-locked at the fundamental frequencies of the laser cavities in the order of only tens of megahertz. Such low repetition rate is only promising for applications where higher peak power is required such as optical imaging rather than transmission systems where high repetition rate is more crucial for larger information capacity. Moreover, the pulses are self-started without any external clock. Therefore the pulses are not precisely equally spaced in the time domain in passive mode-locking systems ${ }^{4}$, which may also have some harmful effect on signal transmission process. In optical transmission applications, actively mode-locked laser is preferred. It can be operated under much higher repetition rate in the order of gigahertz and the pulse train is precisely synchronized with an external master clock. Besides the fiber lasers, the photonic crystal fibers (PCFs) have great potential to participate as the transmission fibers in $1-\mu \mathrm{m}$ band, which make transmission applications in this new band more attractive and feasible. PCFs can be manufactured to be single-mode for any wavelength ${ }^{5}$ in that range with low loss. A demonstration of a tunable 10-GHz, around 10.5-ps actively mode-locked $\mathrm{Yb}$ fiber laser has been reported ${ }^{6}$. The original tuning range of the mode-locked fiber laser was $1054 \mathrm{~nm}$ to $1080 \mathrm{~nm}$. After spectrum broadening by utilizing the nonlinearity of the PCF, the tuning range of this laser was slightly broadened to be from $1051 \mathrm{~nm}$ to $1085 \mathrm{~nm}$. Another work utilized the dispersion character of PCF by inserting it into the cavity to manage the net dispersion to anomalous dispersion regime and also compensated the chirp outside the cavity ${ }^{7}$. With the soliton effect, a tunable 10-GHz pulse 
train with short pulsewidth of $2.5 \mathrm{ps}$ was obtained in $1.1 \mu \mathrm{m}$. In this paper, we experimentally demonstrated an actively mode-locked $\mathrm{Yb}$ fiber laser in the shorter wavelength range at around $1030 \mathrm{~nm}$, which more closely matched with the peak of the emission cross section of YDF. Without any additional dispersion management or chirp compensation, a 10-GHz, around 13-ps pulse train tunable from $1023.5 \mathrm{~nm}$ to $1053.3 \mathrm{~nm}$ was achieved.

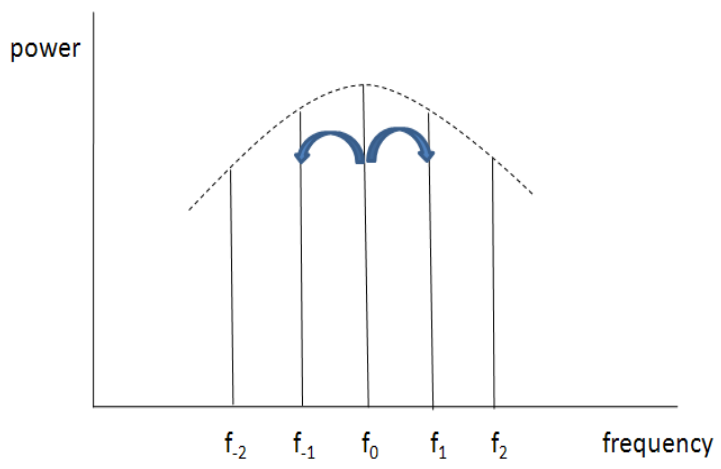

Fig. 1. Schematic diagram of mode locking in the frequency domain.

\section{PRINCIPLE}

Under normal circumstances, there are multiple longitudinal modes oscillating in the fiber laser cavity simultaneously. The phase and amplitude relationships between them are totally random, which correspondingly result in random intensity variation in the time domain. In order to get a stable output, mode-locking technologies are introduced, both active and passive Longitudinal modes are equally spaced by the free spectral range (FSR) $f_{c}=c /(n L)$ where $c$ is the light speed in the vacuum, $n$ is the refractive index, and $L$ is the cavity length. In an all-fiber setup, the total length is usually in the order of tens of meters to incorporate all the components with fiber pigtails. So $f_{c}$ is in the order of tens of megahertz. It is technically challenging to find a filter with a bandwidth narrow enough to filter out a single-longitudinal-mode (SLM) and the number of the longitudinal modes inside the cavity is huge. In actively mode-locked laser, an amplitude modulator (AM) is inserted in the cavity to modulate the loss of the cavity. According to the Fourier transform, such modulation at the same time produces two sidebands to each longitudinal mode at the spacing of the modulation frequency fm and these sidebands are driven in-phase. When the $f_{m}$ coincides with the multiples of $f_{c}$ of the cavity, the sidebands overlap with the existing longitudinal modes in the cavity. These modes are modulated with the same modulation frequency by AM and therefore higher order sidebands are produced as shown in Fig. 1. Finally, the phases of these multiple longitudinal modes are locked and the oscillation inside the cavity evolves into pulse radiation. By tuning the optical bandpass filter (OBPF) inside the cavity, the pulsing wavelength can be easily tuned. According to the analytical theory of active mode-locking established by Siegman and Kuizenga8, the output pulse shape is almost Gaussian. When we simplify the active mode-locking cavity by only considering the optical filter and intensity modulation inside the cavity ${ }^{9}$, the chirp-free pulsewidth can be estimated by 


$$
\tau=\frac{1}{2 \pi} \sqrt[4]{\frac{1}{m f_{m}^{2} B_{f}^{2}}}
$$

where $\tau$ is the pulsewidth at the 1/e intensity point. The full width at half maximum (FWHM) pulsewidth $T_{F W H M}$ should be $T_{F W H M}=1.665 \tau$. Modulation depth $m$ is the ratio of the voltage $V p-p$ to $V \pi$. $B_{f}$ is the $1 / \mathrm{e}$ bandwidth of the optical filter's bandwidth and $B_{f}=v_{F W H M} / 1.665$.

\section{EXPERIMENT}

The experimental setup is illustrated in Fig. 2. The gain in the laser cavity was provided by a 30-cm long YDF. The YDF is highly-doped and the absorption rate at $974 \mathrm{~nm}$ is around $1000 \mathrm{~dB} / \mathrm{m}$. Pump source at $976 \mathrm{~nm}$ with the maximum pump power of $400 \mathrm{~mW}$ was launched into the cavity through a 980/1060 wavelength-division multiplexing (WDM) coupler. The $3-\mathrm{dB}$ bandwidth of the $\mathrm{OBPF}$ is around $1.1 \mathrm{~nm}$. A polarization controller (PC) aligned the state-of-polarization (SOP) of the light with the transmission axis of the AM to minimize the insertion loss. RF signal applied to the AM was sinusoidal signal at around $10 \mathrm{GHz}$. An isolator was inserted to ensure the unidirectional operation of the cavity. The output from the 90/10 coupler was further amplified by an YDFA and then analyzed on digital communication analyzer (DCA) and electrical spectrum analyzer (ESA).

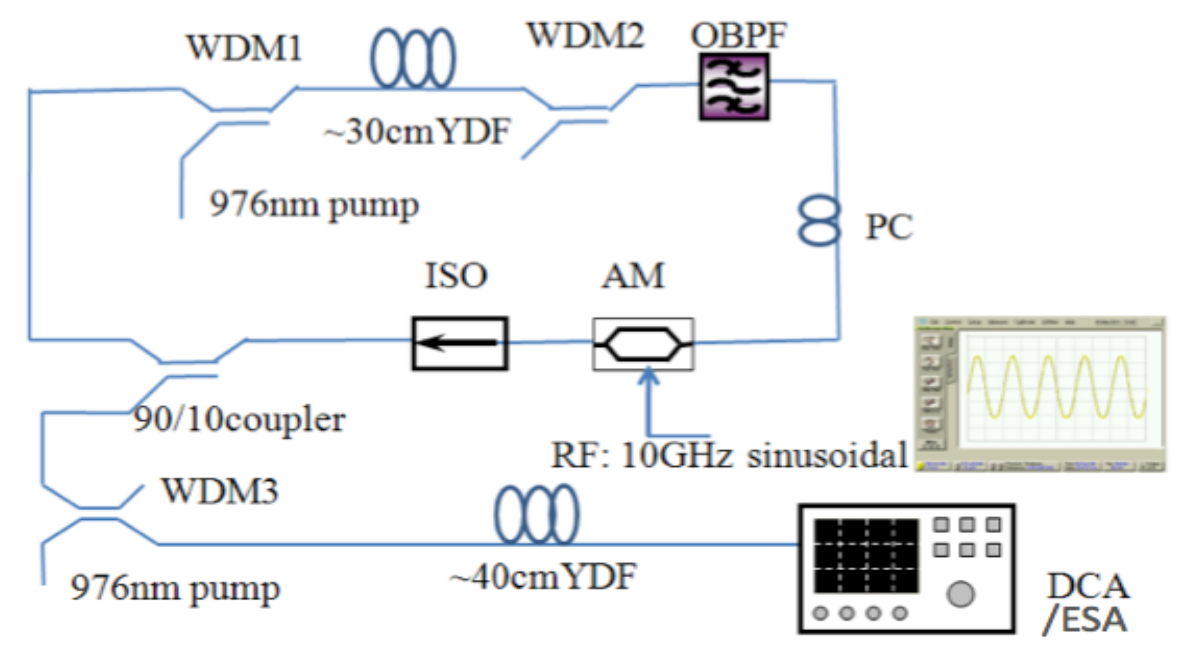

Fig. 2. Experimental setup of the $10-\mathrm{GHz}$ active mode-locking fiber laser. Abbreviations are defined in text.

Since lights with different wavelengths travel at different speeds inside the optical fiber, each time we tuned the central wavelength of the OBPF, the modulation frequency needed to be finely adjusted to match the harmonics of the FSR of the cavity at that specific wavelength as shown in Fig.3 (a). The tuning range was from $1023.5 \mathrm{~nm}$ to $1053.3 \mathrm{~nm}$ (around $30 \mathrm{~nm}$ ). The modulation frequency changed linearly with the operation wavelength. From the waveforms on DCA, the pulse quality decreased slightly as the filter was tuned away from the emission peak of YDF at around $1030 \mathrm{~nm}$. Fig. 3 (b) - (e) show the waveforms of the pulse train with wavelengths in the middle and at the edges of the tuning range, respectively. When the pulse was at $1032.07 \mathrm{~nm}$, the pulsewidth was measured to be $13.0 \mathrm{ps}$ from the DCA (the optical bandwidth of the DCA is $53 \mathrm{GHz}$ ) and the FWHM of the spectrum was $1.12 \mathrm{~nm}$. The modulation frequency was about $9.2 \mathrm{GHz}$. When we took the modulation depth $m$ as 0.5 , the calculated pulsewidth at $1032.07 \mathrm{~nm}$ was $7.3 \mathrm{ps}$ by following 


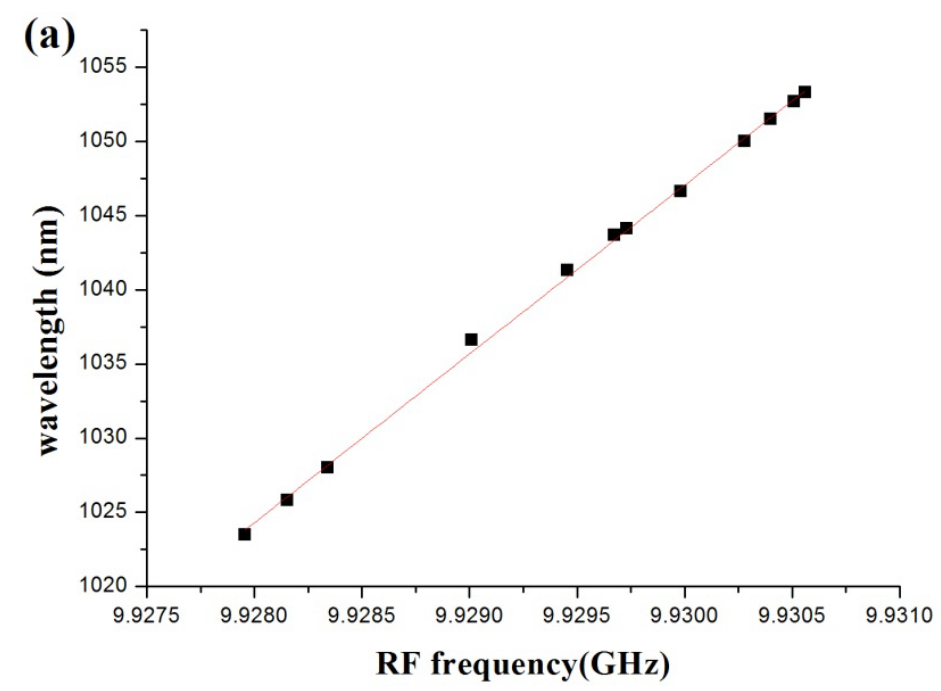

(b)
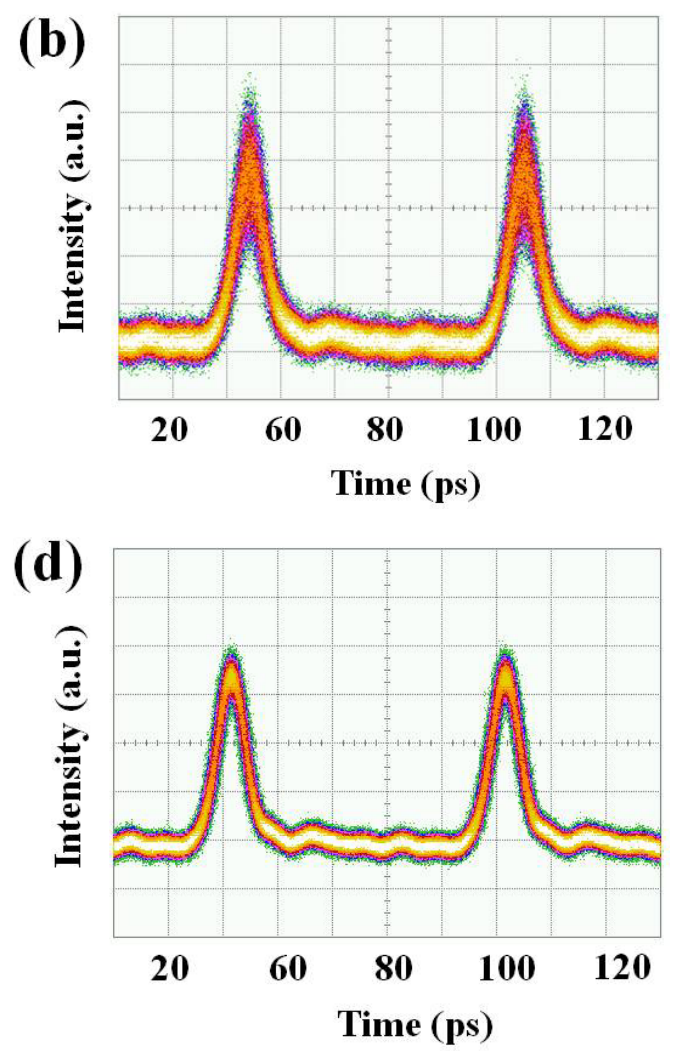

(c)

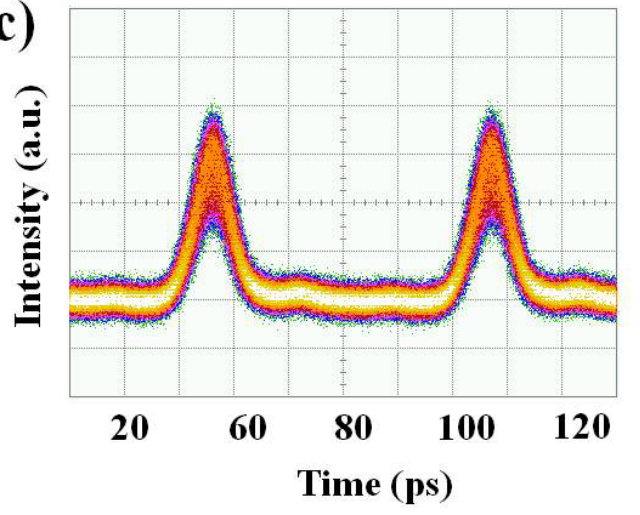

(e)

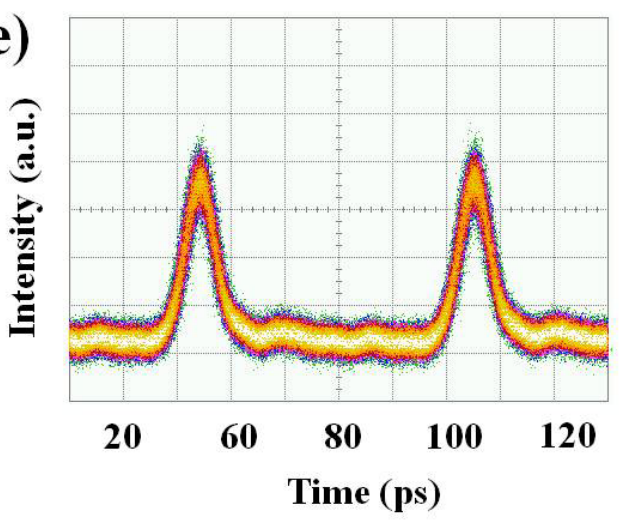

Fig. 3. (a) Tunable wavelength versus the modulation frequency;

Waveforms at (b) $1053.3 \mathrm{~nm}$; (c) $1023.5 \mathrm{~nm}$; (d)1032.1 nm; (e) $1040.9 \mathrm{~nm}$.

equation (1), which corresponded to an optical bandwidth of more than $40 \mathrm{GHz}$. The measured value, which was $13.0 \mathrm{ps}$ from the DCA, was larger than the numerical one due to the limitation of the electrical bandwidth of the photodetector. Moreover, the laser cavity was operated under normal dispersion regime due to the high value of normal material dispersion at wavelengths shorter than $1.1 \mu \mathrm{m}$ in silica glass. The pulse inside the cavity was not chirp-free as assumed in 
equation (1). The actual pulsewidth was supposed to be slightly larger than the numerical value since the numerical model did not take the broadening effect of the dispersion into account. Therefore, the pulsewidth of the output can be further shortened with intracavity dispersion management or chirp compensation outside the cavity ${ }^{7}$. Fig. 4 shows the electrical spectra of the output pulse. The side-mode suppression ratio was more than $50 \mathrm{~dB}$. The active mode-locking setup could be affected by the environmental disturbances such as the temperature since the round trip time of the intracavity pulses would drift and misalign with the external clock. The side-mode suppression ratio could be further improved by adding some stablizing techniques like temperature control and phase-locked loop ${ }^{7}$.
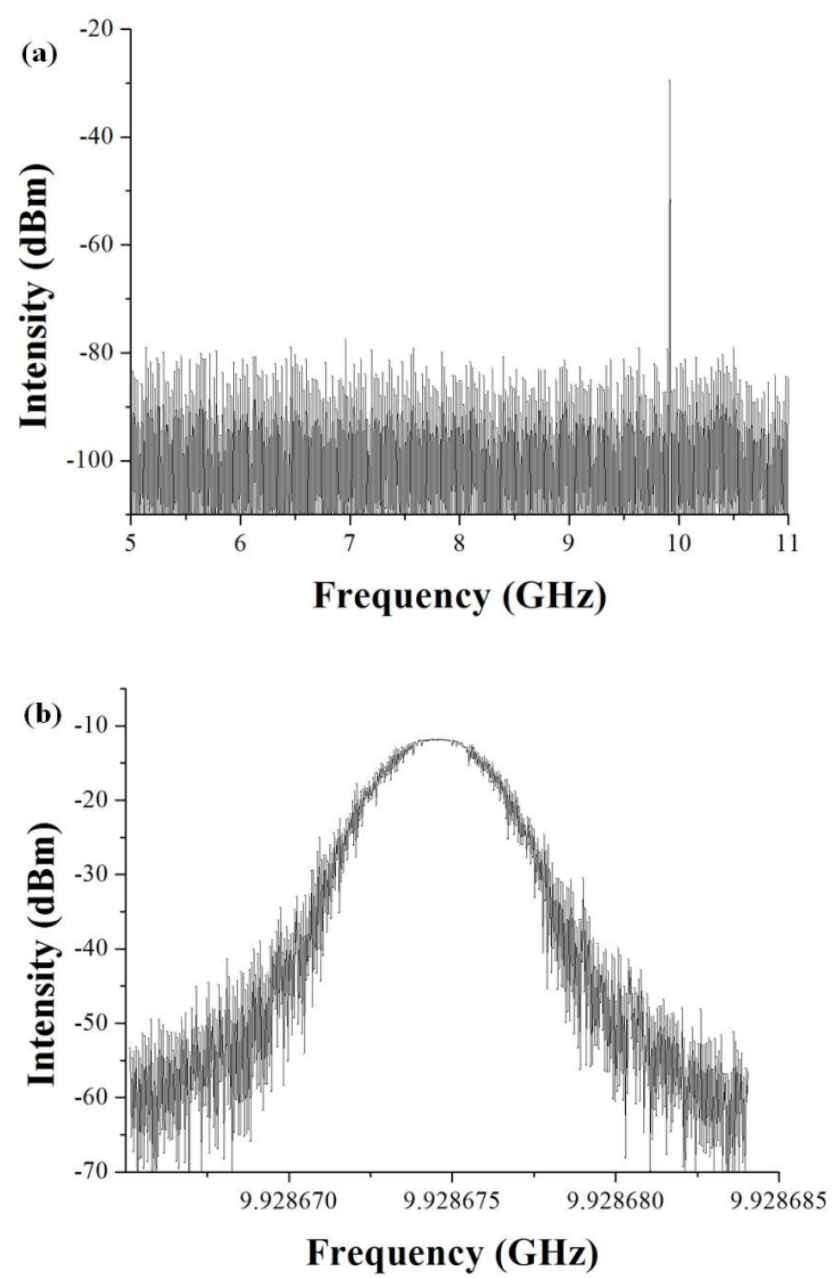

Fig. 4. (a) Electrical spectrum of the pulse at $1032.07 \mathrm{~nm}$ on ESA; (b) The details of the mode at $9.929 \mathrm{GHz}$.

\section{CONCLUSION}

In this paper, we demonstrated a 10-GHz actively mode-locked all-fiber laser with a tuning range from $1023.5 \mathrm{~nm}$ to $1053.3 \mathrm{~nm}$ over a range of about $30 \mathrm{~nm}$. The pulsewidth was around $13 \mathrm{ps}$ without any dispersion management or chirp compensation. More than 50-dB side-mode suppression ratio was achieved. The pulse quality can be further improved by 
adding some stabilization technologies and some dispersion management components. Such kind of optical source is attractive in terms of optical transmission applications in 1- $\mu \mathrm{m}$ band. Its operation regime is closer to the emission peak of YDF at around $1030 \mathrm{~nm}$ compared with the previous works ${ }^{6,7}$. Larger emission cross section of YDF in this shorter wavelength range may bring higher power conversion efficiency in the amplification and transmission processes. Moreover, since there is no gap between the tunable wavelength ranges in our work and the previous work ${ }^{6}$, a widely continuously tunable short pulse source with high repetition rate covering almost the whole $1-\mu \mathrm{m}$ band is possible in the future work.

\section{ACKNOWLEDGEMENTS}

The work in this paper was partially supported by grants from the Research Grants Council of the Hong Kong Special Administrative Region, China (projects HKU 7179/08E and HKU7183/09E).

\section{REFERENCES}

[1] D. J. Richardson, J. Nilsson and W. A. Clarkson, "High power fiber lasers: current status and future perspectives," $J$. Opt. Soc. Am. B 27, B63-B92 (2010).

[2] A. V. Ivanenko, S. M. Kobtsev and S. V. Kukarin, "Femtosecond ring all-fiber Yb laser with combined wavelength-division multiplexer-Isolator,", Laser Physics, 20(2), 344-346 (2010).

[3] L. A. Gomes, L. Orsila, T. Jouhti and O. G. Okhotnikov, "Picosecond SESAM-based ytterbium mode-locked fiber lasers," IEEE J. Quantum Electron., 10(1), 129-136 (2004).

[4] S. Gray, and A. B. Grudinin, "Soliton fiber laser with a hybrid saturable absorber," Opt. Lett., 21, 207-209 (1996).

[5] T. A. Birks, J. C. Night and P. St. J. Russell, “ Endlessly single-mode photonic crystal fiber,” Opt. Lett., 22, 961-963 (1997).

[6] T. Yamamoto, K. Kurokawa, K. Tajima and T. Kurashima, "1.0 $\mu \mathrm{m}$ band, 4.22- THz spectral bandwidth WDM signal pulse source using photonic crystal fibers,” OFC, OWD3, 2008.

[7] K. Koizumi, M. Yoshida, T. Hirooka and M. Nakazawa, "A $10 \mathrm{GHz} 2.5$ ps regeneratively mode-locked Yb fiber laser in the $1.1 \mu \mathrm{m}$ band," CLEO, CMBB3, 2011.

[8] D. I. Kuizenga and A. E. Siegman, "Modulator frequency detuning effects in the FM mode-locked laser," IEEE J. Quantum Electron., QE-6, 803-808 (1970).

[9] Y. H. Li, C. Y. Lou, M. Han and Y. Z. Gao, "Detuning characteristics of the AM mode-locked fiber laser," Optical and Quantum Electronics, 33, pp. 589-597, Jul. 2001. 